\title{
A structured filter for Markovian switching systems
}

\author{
Abdelfettah Hocine ${ }^{\mathrm{a}}$, Mohammed Chadli ${ }^{\mathrm{b}, *}$ and Hamid Reza Karimi ${ }^{\mathrm{c}}$ \\ ${ }^{a}$ LESI, Centre Universitaire de Khemis Miliana, Khemis Miliana, Algeria; ${ }^{b}$ MIS, Université de Picardie Jules Verne, Amiens, France; \\ ${ }^{c}$ Department of Engineering, University of Agder, Grimstad, Norway
}

(Received 28 July 2013; accepted 22 December 2013)

\begin{abstract}
In this work, a new methodology for the structuring of multiple model estimation schemas is developed. The proposed filter is applied to the estimation and detection of active mode in dynamic systems. The discrete-time Markovian switching systems represented by several linear models, associated with a particular operating mode, are studied. Therefore, the main idea of this work is the subdivision of the models set to some subsets in order to improve the detection and estimation performances. Each subset is associated with sub-estimators based on models of the subset. In order to compute the global estimate and subset probabilities, a global estimator is proposed. Theoretical developments based on a hierarchical decision, leading to more efficiency in detection and state estimation, are proposed. Naturally, these results can be used for fault detection and isolation, using the activation probabilities of operating modes. These results are applied to detect switches in the centre of gravity for vehicle roll dynamics.
\end{abstract}

Keywords: Markovian switching system; multiple model estimation; active mode detection; vehicle roll dynamic

\section{Introduction}

Multiple model estimation methods for Markovian switching systems, which include an Interacting Multiple Model (IMM) and Generalized Pseudo-Bayesian (GPB) algorithm, need a priori knowledge about the mode transition proba-bilities represented by a Markov chain (Bar-Shalom, Chang, \& Blom, 1989; Dong, Wang, \& Gao, 2013; Dong, Wang, Ho, \& Gao, 2011; Hocine, Chadli, Maquin, \& Ragot, 2008; Karimi, 2011; Magill, 1965; Wang, Liu, \& Liu, 2010; Wang, Shen, \& Liu, 2012; Wei, Wang, Shen, \& Li, 2011; Zhang, \& Li, 1998). Traditional methods are based on the use of the Kalman filter associated with each model. In or-der to improve algorithm performances, several studies us-ing techniques like an extended Kalman filter (Boers \& Driessen, 2005) and a finite memory observer (Hocine, Maquin, \& Ragot, 2005) have been reported in the lit-erature. The use of this estimator should be theoretically justified to ensure suboptimality of the methods. The con-struction of the models set is very important and directly affects the results of the estimation (Li \& Bar-Shalom, 1996) and influences the quality of detection. The qual-ity of detection can decrease when the set size increases (Li \& Bar-Shalom, 1996; Qu, Pang, \& Li, 2009). To solve this problem, the authors of Li and Bar-Shalom (1996), Li (2000), and Li, Zhao, and Li (2005) introduced a con-cept of variable structure multiple model estimator with a variable model set. Then, the number of models and there-fore the dispersion probability is reduced. These results

\footnotetext{
${ }^{*}$ Corresponding author. Email: mohammed.chadli@u-picardie.fr
}

have been recently applied to manoeuvring target tracking (Ho, 2011). These works are based on using, at each time, a subset of models instead of a whole set using switching logic. Unfortunately, these methods lead to good results only in the case of known switching sequences. Moreover, for an arbitrary switching, it is hard to apply this technique. In this paper, a method based on the whole set of models is presented. The basic idea is to divide the set of models into some subsets. Compared with previous works, the proposed method gives systematic ways to compute Markov chains, state estimates and probability of the subsets. The study gives a theoretical procedure to construct a new multiple model estimator. The developed structure is adapted to a parallel computation for greater speed in real-time working.

The rest of the paper is organised as follows. In Sections 2 and 3, procedures of computing transition matrices proba-bilities between models of a subset and between subsets are exposed, respectively. Then, a structured filter for Marko-vian switching systems is defined and detailed in Section 4. Afterwards, a detection methodology of active mode is given in Section 5, using the result of the structured fil-ter. Finally, to show the benefit of the proposed algorithm compared to the interacting multiple model (IMM) algo-rithm, an application of vehicle roll dynamics is given in order to detect changes in the centre of gravity $(\mathrm{CG})$.

Notation: Throughout the paper, the following notations are used. $\pi_{i j}=P\left\{M_{i} \mid M_{j}\right\}$ denotes the transition probabilities between the models $M_{i}$ and $M_{j}, \mu^{s_{j}}(k)$ is the activation 
probabilities of models belonging to the subset $s_{j}, \mu^{s_{j}}(k)$ is the activation probabilities of the subset $s_{j}$, and $\mu_{i}(k)$ is the activation probability of the model $j$ and $I_{r}=\{1,2, \ldots, r\}$.

\section{Problem statement}

We consider a system represented by a set of models $M=$ $\left\{M_{1}, M_{2}, \ldots, M_{r}\right\}$, where $r$ is a model number. When $M_{i}$ is active, the dynamics of the system is given by the following equation:

$$
\left\{\begin{array}{l}
x(k)=A_{i} x(k-1)+B_{i} u(k-1)+G_{i} w(k-1) \\
z(k)=C_{i} x(k)+v(k)
\end{array}\right.
$$

where $x$ is the state vector, $z$ is the noisy measurement vector, $i$ is the system mode index which denotes the active mode, and $w$ and $v$ are noise sequences, with normal distributions, on mode-dependent process and measurement, respectively. The system mode sequence is assumed to be a first-order homogeneous Markov chain with the following Markovian transition matrix:

$$
\Pi=\left[\begin{array}{ccc}
\pi_{11} & \cdots & \pi_{1 r} \\
\vdots & \ddots & \vdots \\
\pi_{r 1} & \cdots & \pi_{r r}
\end{array}\right]
$$

Division of the set $M$ into some subsets $s_{i}$ gives a set of subsets $S=\left\{s_{1}, s_{2}, \ldots, s_{n}\right\}$, where $n$ represents the number of subsets and $s_{i} \bigcap s_{j}=\emptyset, \forall i \neq j \in I_{n}$, and $\bigcup_{i=1}^{n} s_{i}=$ $M$.

Each subset is associated with a sub-estimator based on the models of the subset, $s_{i}=\left\{M_{\left\{s_{j}, 1\right\}}, M_{\left\{s_{j}, 2\right\}}, \ldots\right.$, $\left.M_{\left\{s_{j}, r_{j}\right\}}\right\}$, where $r_{j}$ represents a model number of $s_{i}$ and $\left\{s_{j}, j\right\}$ is the model index in the set $M$. Afterwards, a global estimator is constructed in order to collect the estimations and probabilities of each sub-estimator to compute the global estimate and probabilities of subsets.

To construct a global estimator, we need to compute the Markovian transition matrix $\Pi_{s}$, which represents transition probabilities between two subsets $s_{i}$ and $s_{j}$. To get $\Pi_{s}$, we use the Markovian transition matrix $\Pi$, assumed to be known.

\section{Markovian transition matrix}

To develop the sub-estimator and the global estimator, we should compute the transition matrix probabilities of each subset and transition matrix related to the set of subsets. Using the Markovian switching matrix of the set of whole model $\Pi$, we can compute the transition matrix $\Pi_{s}$ from a subset to another and the matrices $\Pi^{s_{i}}, i \in I_{n}$ governing the transitions from a model to another in the same subset $s_{i}$.

\subsection{Markovian matrix of subsets transition}

Considering the stochastic process of switching as stationary, we can now write

$$
\gamma=\Pi \gamma, \quad \gamma_{j}=\sum_{i=1}^{r} \gamma_{i} \pi_{i j}, \quad j \in I_{r},
$$

where $\gamma=\left[\gamma_{1}, \gamma_{2}, \ldots, \gamma_{r}\right]$ is the vector of probabilities $\gamma_{j}$ of each model $M_{i}$ with $\Sigma_{i=1}^{r} \gamma_{i}=1$. The $\gamma_{j}$ allows us to compute Markovian matrix of subsets transition using the Markovian transition matrix $\Pi$.

The transition probability from a subset to another is given by the following equation:

$$
\pi_{s_{i} \mid s_{j}}=\frac{\sum_{l=1}^{r_{i}} \gamma_{l} \sum_{h=1}^{r_{j}} \pi_{\left\{s_{i}, l\right\}\left\{s_{j}, h\right\}}}{\sum_{l=1}^{r_{i}} \gamma_{l}} \quad i, j \in I_{n},
$$

where $\pi_{s_{i} \mid s_{j}}$ is the transition probability from the subset $s_{i}$ to subset $s_{j}$ and $\pi_{\left\{s_{i}, l\right\}\left\{s_{j}, h\right\}}$ is the transition probability from the model $l$ belonging to the subset $s_{i}$ to the model $h$ belonging to the subset $s_{j}$. These probabilities are provided directly by the matrix $\Pi$.

\subsection{Markovian transition matrix of subset models}

In order to construct the sub-estimators, related to the subsets, we need to compute transition matrices $\Pi^{s_{j}}$. From the matrix $\Pi$, we directly get

$$
\pi_{l h}^{s_{j}}=\frac{\pi_{l h}}{\sum_{i=1}^{r_{j}} \pi_{\left\{s_{j}, l\right\}\left\{s_{j}, i\right\}}} \quad l, h \in I_{r_{j}},
$$

where $\pi_{l h}^{s_{j}}$ is the transition probability from model $l$ to model $h$ belonging to subset $s_{j}$.

\section{Estimation procedure}

The proposed procedure consists of dividing the set of models $M$ into subsets $s_{i}$ and proposing a sub-estimator for each subset $s_{i}$. These sub-estimators can be either IMM or GPB estimators or other kinds of multiple model Bayesian estimators. The multiple model estimator gives the state estimate, its variance and the activation probability of each model belonging to the subset. Thereafter, the construction of the estimator is based on two levels of estimation (sub-estimator and global estimator).

\subsection{Sub-estimator}

At time $k$, the inputs of the sub-estimator are measurements $z(k)$, and the estimate is given by the global estimator $\hat{x}(k-$ $1 \mid k-1)$ and its variance $P(k-1 \mid k-1)$ at time $k-1$ (Figure 1). These inputs are then used as the input for $r_{i}$ 


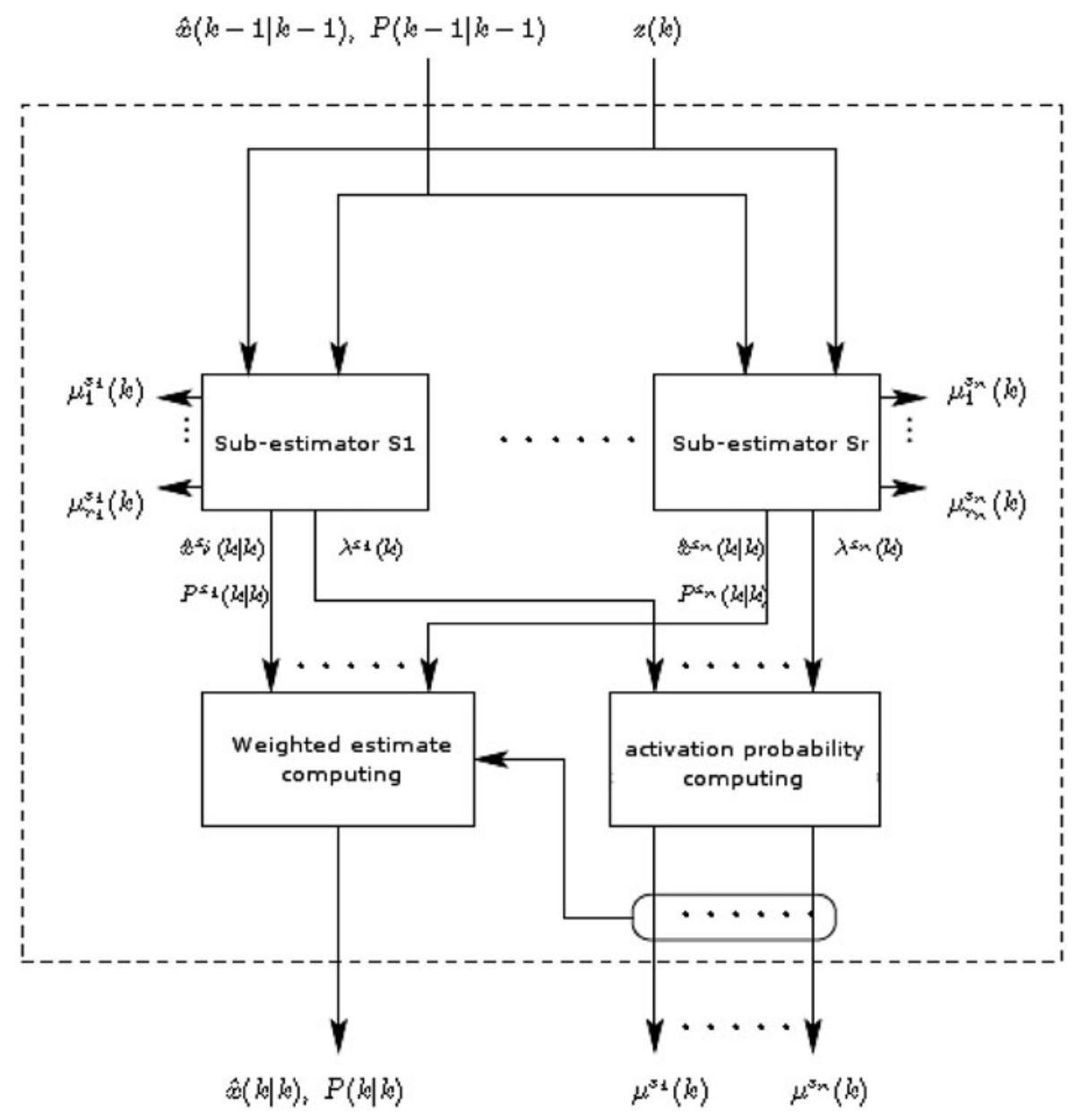

Figure 1. Sub-estimator.

different Kalman filters associated with the subset $s_{i}, i \in I_{n}$, where $n$ is the number of subsets. As the output of filters we have the estimates $\hat{x}_{j}^{s_{i}}(k \mid k)$ for $j \in I_{r_{i}}$, where $r_{i}$ is the number of models in the subset $i$ and the associate variances $P_{j}^{s_{i}}(k \mid k)$.

Likelihood function. The likelihood function corresponding to the $j$ th Kalman filter of the $i$ th subset $s_{i}$ is given by

$$
\lambda_{j}^{s_{i}}(k)=p\left[z(k) \mid Z^{k-1}, M_{\left\{s_{i}, j\right\}}\right]
$$

where $j \in I_{r_{i}}$ is computed using the estimate $\hat{x}_{i}^{s_{j}}(k-1 \mid k-1)$ and its variance

$$
\begin{aligned}
\lambda_{j}^{s_{i}}(k)= & p\left[z(k) \mid M_{\left\{s_{i}, j\right\}}, \hat{x}(k-1 \mid k-1),\right. \\
& P(k-1 \mid k-1)] .
\end{aligned}
$$

Considering $w$ and $v$ with a normal distribution, we have the following likelihood function:

$$
\lambda_{j}^{s_{i}}(k)=\mathbf{N}\left[v_{j}^{s_{i}}(k) ; 0, S_{j}^{s_{i}}(k)\right]
$$

where $\quad v_{j}^{s_{i}}(k)=z(k)-C_{j}^{s_{i}}\left(A_{j}^{s_{i}} \hat{x}(k-1 \mid k-1)+B_{j}^{s_{i}} u\right.$ $(k-1))$ and its variance $S_{j}^{S_{i}}(k)$, with $B_{j}^{S_{i}}$, and $C_{j}^{S_{i}}$ are associated with the $j$ th model of the subset $s_{i}$.

Update activation probabilities of models. Like the GPB1 algorithm, the use of Bayes' formula allows the following development:

$$
\mu_{j}^{s_{i}}(k)=\frac{1}{c_{i}^{s_{i}}(k)} \lambda_{j}^{s_{i}}(k) \sum_{l=1}^{r_{i}} \pi_{l j}^{s_{i}} \mu_{l}^{s_{i}}(k-1),
$$

where $\mu_{i}^{s_{j}}(k)$ is the activation probability of the model $j$, at time $k$, belonging to the subset $s_{j}$ and $c_{j}^{s_{j}}$ is a normalisation constant given by

$$
c_{i}^{s_{j}}(k)=\sum_{j=1}^{r_{i}} \lambda_{j}^{s_{i}}(k) \sum_{l=1}^{r_{i}} \pi_{l j}^{s_{i}} \mu_{l}^{s_{i}}(k-1) .
$$




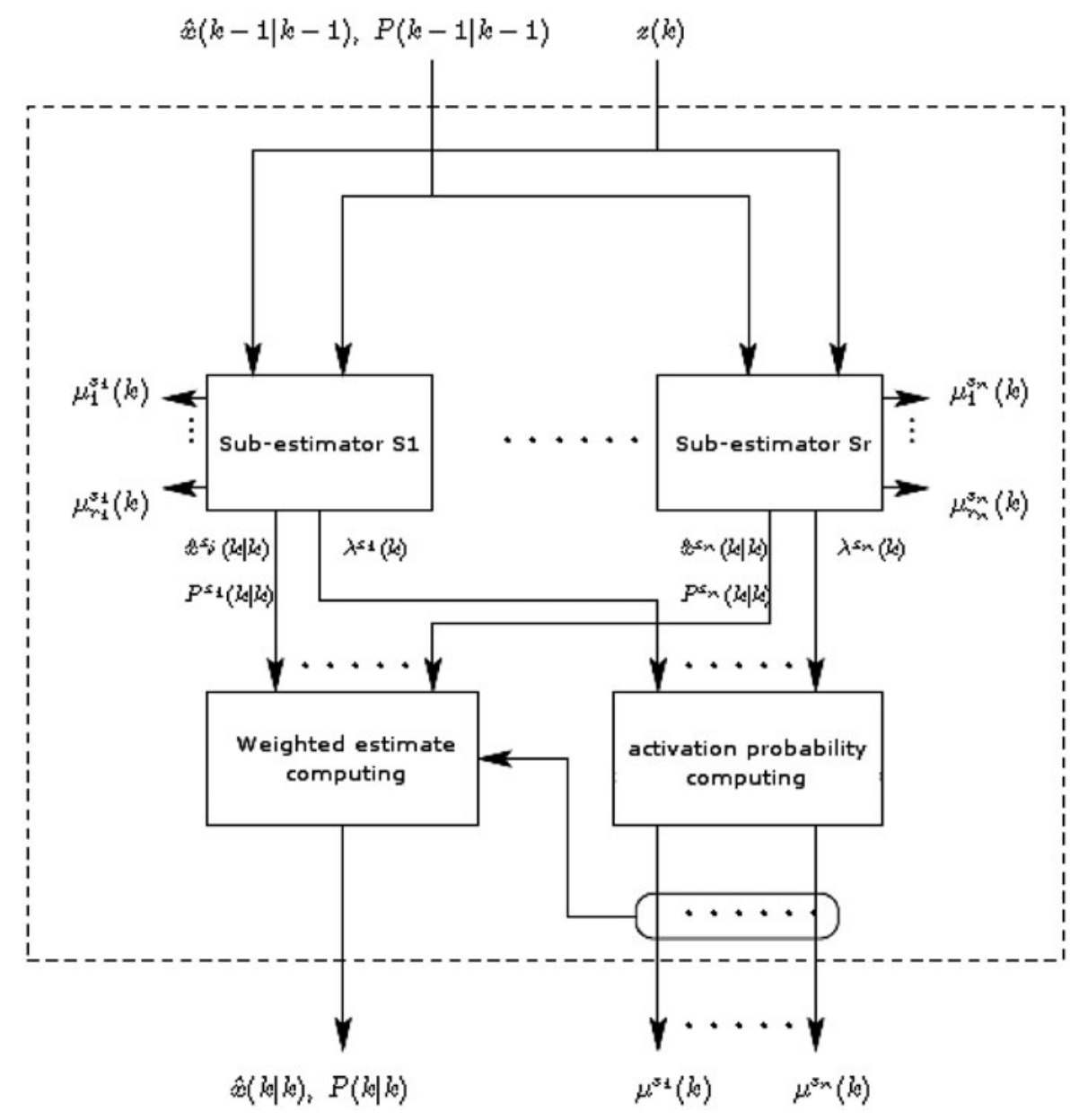

Figure 2. Global estimator.

The weighted estimate of subset $s_{j}$. The weighted estimate is given by

$$
\hat{x}^{s_{i}}(k \mid k)=\sum_{j=1}^{r_{i}} \mu_{j}^{s_{i}}(k) \hat{x}_{j}^{s_{i}}(k \mid k)
$$

and its variance

$$
\begin{aligned}
P^{s_{i}}(k \mid k)= & \sum_{j=1}^{r_{i}} \mu_{i}^{s_{i}}(k)\left\{P_{j}^{s_{i}}(k \mid k)+\left[\hat{x}_{j}^{s_{i}}(k \mid k)\right.\right. \\
& \left.\left.-\hat{x}^{s_{i}}(k \mid k)\right]\left[\hat{x}_{j}^{s_{i}}(k \mid k)-\hat{x}^{s_{i}}(k \mid k)\right]^{\prime}\right\}
\end{aligned}
$$

where the estimate $\hat{x}_{j}^{s_{i}}(k \mid k)$ is an output of the $j$ th Kalman filter related to the $j$ th model of the subset $s_{i}$.

Each sub-estimator gives a weighted estimate $\hat{x}^{s_{i}}(k \mid k)$, and the activation probabilities $\mu_{i}^{s_{j}}(k), \quad i \in I_{r_{j}}$, of models belonging to the subset $s_{j}$.

The different steps of the sub-estimator are represented in Figure 1. As the input of the sub-estimator, there are the measurement, at time $k$, and the global state $\hat{x}(k-1 \mid k-1)$, which will be defined in the following section. As the output, we have the estimate $\hat{x}^{s_{i}}(k \mid k)$ related to the subset $s_{i}$.

\subsection{Global estimator}

The global estimator structure is similar to the GPB estimator structure. Instead of Kalman filters in the GPB, the $n$ parallel filters in the global estimator are the $n$ subestimators presented above.

The inputs of the global estimator are the measurement $z(k)$ and the estimate at time $k-1, \hat{x}(k-1 \mid k-1)$, and its variance $P(k-1 \mid k-1)$. These inputs are used as the input for each sub-estimator. The global estimator (Figure 2) gives the estimate $\hat{x}(k \mid k)$ at time $k$ and its variance $P(k \mid k)$. During the estimation, we compute the probability activation of the subsets $\mu^{s_{i}}(k), i \in I_{n}$.

Likelihood function. The likelihood function related to the $i$ th sub-estimator is given by

$$
\lambda^{s_{i}}(k)=p\left[z(k) \mid Z^{k-1}, s_{i}\right]
$$




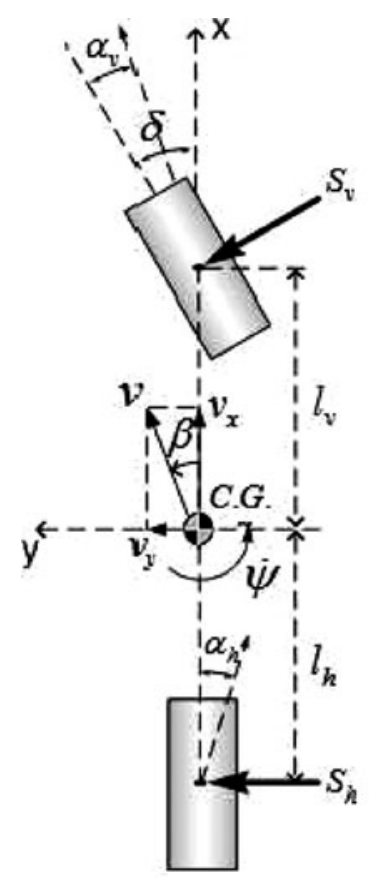

Figure 3. Linear bicycle model with a roll degree of freedom.

with $i \in I_{n}$. It is computed as follows:

$\lambda^{s_{i}}(k)=p\left[z(k) \mid s_{i}, \hat{x}(k-1 \mid k-1), P(k-1 \mid k-1)\right]$.

Then, we have the following likelihood function:

$$
\lambda^{s_{i}}(k)=\mathbf{N}\left[v^{s_{i}}(k) ; 0, S^{s_{i}}(k)\right]
$$

with $v^{s_{i}}(k)=z(k)-C^{s_{i}}(k)\left(A^{s_{i}}(k) \hat{x}(k-1 \mid k-1)+B^{s_{i}}\right.$ $(k) u(k-1))$ and it variance $S^{s_{i}}(k)$.

In a similar way to $\hat{x}^{s_{j}}(k \mid k)$ in $(11), A^{s_{i}}(k), B^{s_{i}}(k)$ and $C^{s_{i}}(k)$ can be represented as

$$
\begin{aligned}
& A^{s_{i}}(k)=\sum_{j=1}^{r_{i}} \mu_{i}^{s_{i}}(k) A_{j}^{s_{i}}, \\
& B^{s_{i}}(k)=\sum_{j=1}^{r_{i}} \mu_{i}^{s_{i}}(k) B_{j}^{s_{i}}, \\
& C^{s_{i}}(k)=\sum_{j=1}^{r_{i}} \mu_{i}^{s_{i}}(k) C_{j}^{s_{i}},
\end{aligned}
$$

where $A_{j}^{s_{i}}, B_{j}^{s_{i}}$ and $C_{j}^{s_{i}}$ are the matrices of the $j$ th model of the subset $s_{i}$. The subset $s_{i}$ is represented by the matrices $A^{s_{i}}(k), B^{s_{i}}(k)$ and $C^{s_{i}}(k)$. These matrices are the sum of matrices related to $s_{i}$ subset models, weighted by the activation probabilities $\mu_{j}^{s_{i}}(k)$.

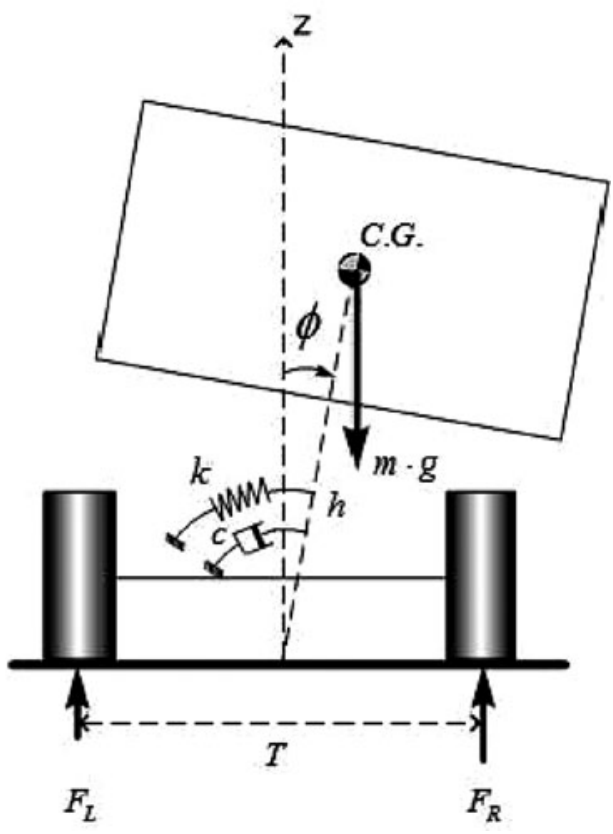

Updating activation probabilities of a subset. The subset activation probabilities are updated as follows:

$$
\mu^{s_{i}}(k)=\frac{1}{c^{s_{i}}(k)} \lambda^{s_{i}}(k) \sum_{j=1}^{n} \pi_{s_{j} \mid s_{i}} \mu^{s_{i}}(k-1)
$$

with a normalisation constant

$$
c^{s_{i}}(k)=\sum_{i=1}^{n} \lambda^{s_{i}}(k) \sum_{j=1}^{n} \pi_{s_{j} \mid s_{i}} \mu^{s_{i}}(k-1) .
$$

Global estimate. From (17) and (11), we have

$$
\hat{x}(k \mid k)=\sum_{i=1}^{n} \mu^{s_{i}}(k) \hat{x}^{s_{i}}(k \mid k) .
$$

To compute the global estimate $\hat{x}(k \mid k)$, we need to have the estimate issues from the sub-estimator related to the subset $s_{i}$ with $i \in I_{n}$ and the associated probabilities.

The variance of $\hat{x}(k \mid k)$ is as follows:

$$
\begin{aligned}
P(k \mid k)= & \sum_{i=1}^{n} \mu^{s_{i}}(k)\left\{P^{s_{i}}(k \mid k)+\left[\hat{x}^{s_{i}}(k \mid k)-\hat{x}(k \mid k)\right]\right. \\
& \left.\times\left[\hat{x}^{s_{i}}(k \mid k)-\hat{x}(k \mid k)\right]^{\prime}\right\} .
\end{aligned}
$$

In summary, the proposed estimation schema uses $r$ Kalman filters related to $r$ operating models. They are divided into $n$ sub-estimators, each containing $r_{j}$ Kalman fil- 
ters. As the output of sub-estimators, we have the estimates $\hat{x}^{s_{i}}$ from Equation (11) and activation probabilities $\mu_{i}^{s_{j}}$ from Equation (9) of models belonging to the subset $s_{i}$. The subestimates are used to compute the global estimate $\hat{x}(k \mid k)$ and activation probabilities $\mu^{s_{j}}(k)$ of the subset $s_{j}$.

\section{Active model detection}

From the proposed estimation procedure, two kinds of probabilities are computed:

- The activation probability $\mu_{j}^{s_{i}}$ of the model $j$ belonging to the subset $s_{i}$. It is computed by considering only the subset containing this model.

- Activation probability $\mu^{s_{i}}$ of the subset $s_{i}$.

Several decision schemas can be performed based on the use of these two kinds of probabilities.

\subsection{Direct decision}

The activation probability of the model $M_{j}=M_{l}^{s_{i}} m_{j} \in s_{i}$ is the product of the activation probability $\mu^{s_{i}}$ of the subset $s_{i}$ with the activation probability of the model in its subset $\mu_{l}^{s_{i}}$ :

$$
\mu_{j}(k)=\mu^{s_{i}}(k) \mu_{l}^{s_{i}}(k) .
$$

At given time $k$, the decision-making about the active mode is directly performed using $\mu_{j}(k)$ as follows: we declare the model $M_{j}$ active if $\mu_{i}(k)$ is the greatest probability activation. There is no decision-making about the active model if $\mu_{i}(k)$ is under a given threshold.

\subsection{Hierarchical decision}

We may consider, at time $k$, two-level decision-making:

(1) Decision on active subset: In the first level, we make a decision about the active subset using activation probabilities $\mu^{s_{i}}(k)$ of the subset $s_{i}$. To decide which subsets are active, we consider the subset with the highest probability $\mu^{s_{i}}(k)$, then the attribute is value 1 , and attribute value 0 to the other activation probabilities of subsets, so we can write. If we have $i$ as $\mu^{s_{i}}(k)>\mu^{s_{j}}(k), \quad j \in I_{n}$, then we impose $\mu^{s_{i}}(k)=1$, else $\mu^{s_{j}}(k)=0$.

(2) Decision on active model: In the second level, we detect the active model using the activation probabilities $\mu_{i}^{s_{j}}(k)$ of the models belonging to the active subset chosen in the first level. Then, the activation probabilities $\mu_{j}(k)$ of the models can be computed by $\mu_{j}(k)=\mu^{s_{i}}(k) \mu_{l}^{s_{i}}(k)$ which is to write $\mu_{j}=\mu_{l}^{s_{i}}(k)$ for $M_{j} \in s_{i}, M_{j}=M_{l}^{s_{i}}$, and $\mu_{j}(k)=0$ for $M_{j} \notin s_{i}$.

From probabilities $\mu_{i}(k)$, we can decide which model is active at time $k$. As in the direct decision, we cannot take a decision (no active model) if probabilities $\mu_{j}(k)$ are
Table 1. Simulation parameters.

\begin{tabular}{|c|c|c|c|}
\hline Parameter & Description & Value & Unit \\
\hline$m$ & Vehicle mass & 1300 & $(\mathrm{~kg})$ \\
\hline$g$ & Gravitational constant & 9.81 & $\left(\mathrm{~m} / \mathrm{s}^{2}\right)$ \\
\hline$V_{x}$ & Vehicle longitudinal speed & 30 & $(\mathrm{~m} / \mathrm{s})$ \\
\hline$\delta$ & Steering angle & & (rad) \\
\hline$J_{x x}$ & $\begin{array}{l}\text { Roll moment of inertia of the } \\
\text { sprung } \\
\text { mass measured at the CG }\end{array}$ & 400 & $\left(\mathrm{~kg} \mathrm{~m}^{2}\right)$ \\
\hline$J_{z z}$ & $\begin{array}{l}\text { Yaw moment of inertia of the } \\
\text { chassis } \\
\text { measured at the CG }\end{array}$ & 1200 & $\left(\mathrm{~kg} \mathrm{~m}^{2}\right)$ \\
\hline$L$ & $\begin{array}{l}\text { Axle separation, such that } L= \\
\quad l_{v}+l_{h}\end{array}$ & & (m) \\
\hline$T$ & Track width & & (m) \\
\hline$l_{v}$ & $\begin{array}{l}\text { Longitudinal CG position } \\
\text { measured } \\
\text { with respect to the front axle }\end{array}$ & 1.2 & (m) \\
\hline$l_{h}$ & $\begin{array}{l}\text { Longitudinal CG position } \\
\text { measured } \\
\text { with respect to the rear axle }\end{array}$ & 1.3 & (m) \\
\hline$h$ & $\begin{array}{l}\text { CG height measured over the } \\
\text { ground }\end{array}$ & 0.7 & (m) \\
\hline$c$ & $\begin{array}{l}\text { Suspension damping } \\
\text { coefficient }\end{array}$ & 5000 & $\left(\mathrm{~kg} \mathrm{~m}^{2} / \mathrm{s}\right)$ \\
\hline$k$ & Suspension spring stiffness & 3600 & $\left(\mathrm{~kg} \mathrm{~m} / \mathrm{s}^{2}\right)$ \\
\hline$C_{v}$ & $\begin{array}{l}\text { Linear tyre stiffness } \\
\text { coefficient for } \\
\text { the front tyre }\end{array}$ & 60,000 & $(\mathrm{~N} / \mathrm{rad})$ \\
\hline$C_{h}$ & $\begin{array}{l}\text { Linear tyre stiffness } \\
\text { coefficient for } \\
\text { the rear tyre }\end{array}$ & 90,000 & (N/rad) \\
\hline$\beta$ & $\begin{array}{l}\text { Sideslip angle at the vehicle } \\
\text { CG }\end{array}$ & & $(\mathrm{rad})$ \\
\hline$\dot{\phi}$ & $\begin{array}{l}\text { Roll angle measured at the roll } \\
\text { centre }\end{array}$ & & $(\mathrm{rad} / \mathrm{s})$ \\
\hline$\phi$ & $\begin{array}{l}\text { Roll rate measured at the roll } \\
\text { centre }\end{array}$ & & $(\mathrm{rad})$ \\
\hline$\Delta t$ & Sampling time & 0.05 & (s) \\
\hline
\end{tabular}

under a given threshold. The hierarchical decision allows the elimination of the effect of the models belonging to the other subsets. This subset is declared not active by the first level of decision. Therefore, the second level uses only the active subset models instead of the whole set of models.

Remark 1: Note that the hierarchical decision can be used for systems where similar models are regrouped (for instance, models around the same operating point, representing the same kind of failure or model of the same size...), whereas the direct decision can be used where the activation probabilities of subsets are very close and where a decision on the active subset cannot be made.

\section{Application to vehicle detection mode}

The developed result is applied for the detection of parameter changes in automobiles. Detection of right parameters is very important, especially in active control design. In- 

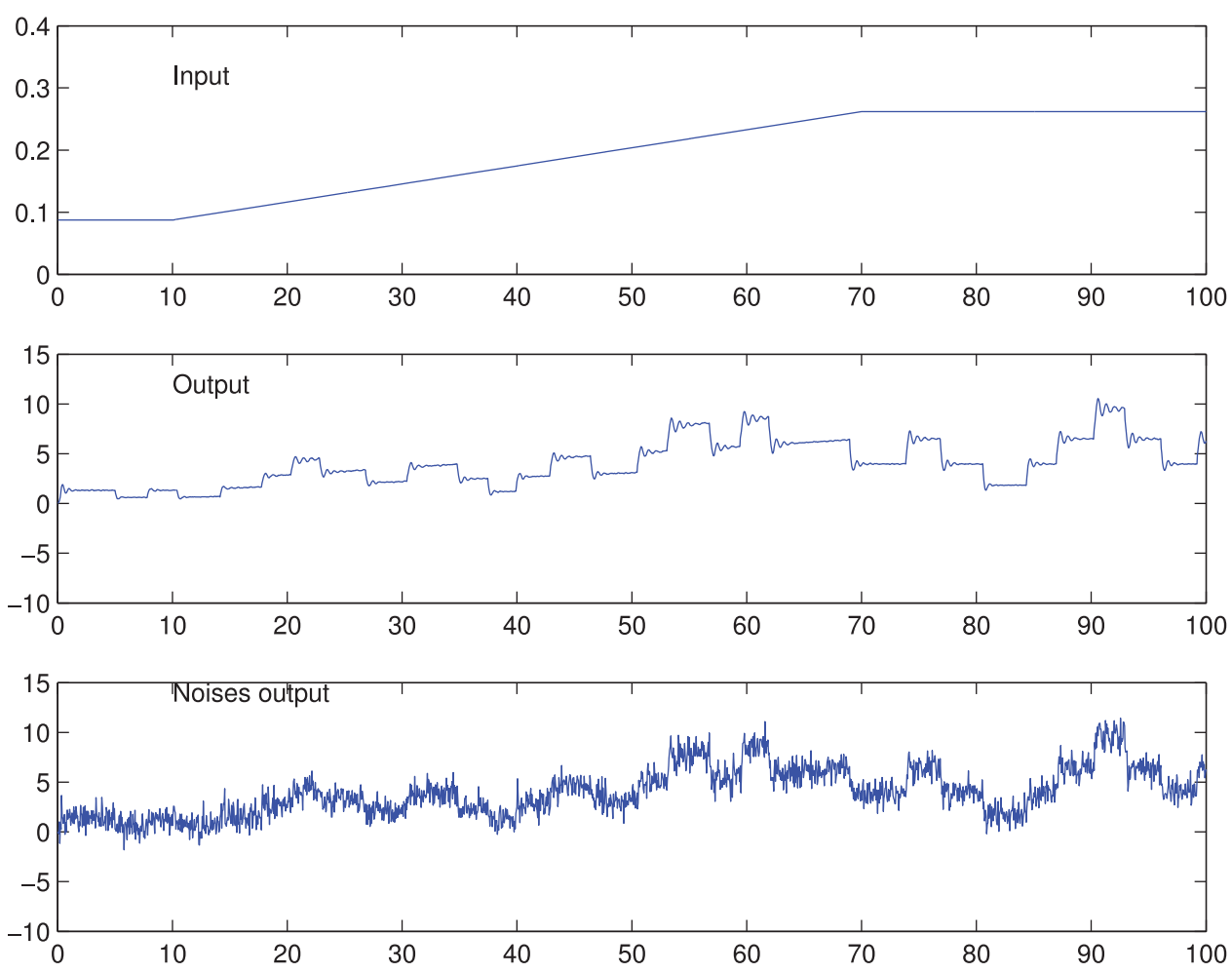

Figure 4. Simulation conditions: steering angle (input $\delta(t)$ ), output $(\phi(t))$ and output with noise.
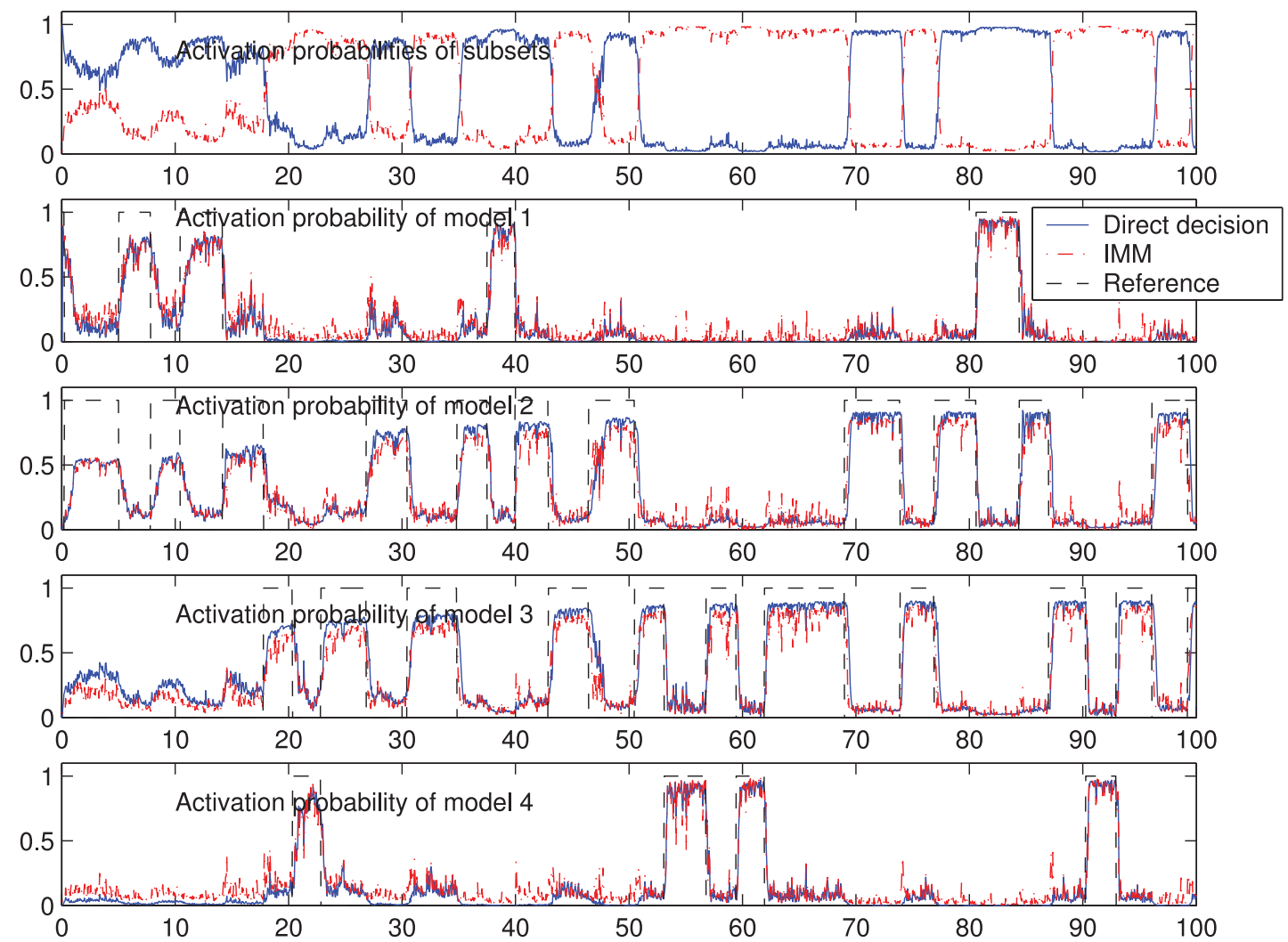

Figure 5. Activation probability (direct method). 

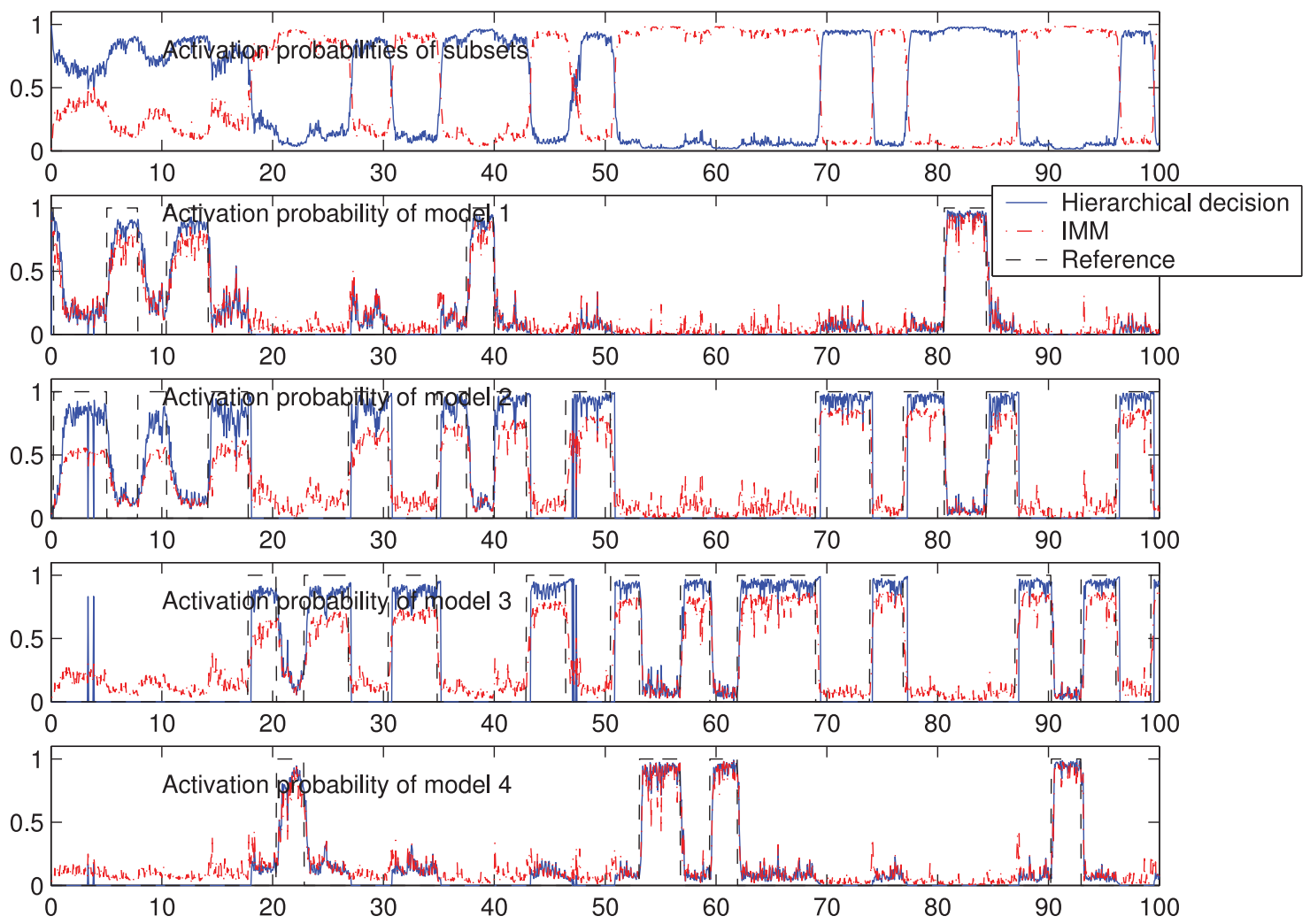

Figure 6. Activation probability (hierarchical decision).

deed, knowing a real value of the CG is a necessary condition to guarantee switching stability in the roll vehicle dynamics subject to rapid changes in CG position. Different vehicle models exist in the literature in nonlinear form and corresponding linear models under some assumptions (Ackermann, Bartelett, Kaesbauer, Sienel, \& Steinhauser, 1993; Bakker, Pacejka, \& Linder, 1989; Dahmani, Chadli, Rabhi, \& El Hajjaji, 2013a; Ryu \& Gerdes, 2004). These nonlinear models describing vehicle lateral and roll dynam-ics consider the nonlinear tyre characteristics (Ackermann et al., 1993; Bakker et al., 1989) or tyre characteristics in their fuzzy form (Dahmani et al., 2013a; Dahmani, Chadli Rabhi, \& El Hajjaji, 2013b). In this paper, we consider a lin-ear bicycle model (Figure 3 ) with a roll degree of freedom where the coupled lateral and roll dynamics assumes that $\delta(t), \phi(t)$ and $\beta(t)$ are small and that all the vehicle mass is sprung (Solmaz, 2007). Using a first-order approximation for the matrix exponentials, the discrete time equivalent of the system can be expressed as follows (Solmaz, Shorten, Wulff, \& O’Cairbre, 2008):

$$
\begin{aligned}
& x(k+1) \\
& =\left[\begin{array}{cccc}
1-\frac{\sigma}{m V_{x}} \frac{J_{x e q}}{J_{x x}} \Delta t & \left(\frac{\rho}{m V_{x}^{2}} \frac{J_{x e q}}{J_{x x}}-1\right) \Delta t & -\frac{h c}{J_{x x} V_{x}} \Delta t & \frac{h(m g h-k)}{J_{x x} V_{x}} \Delta t \\
\frac{\rho}{J_{z z}} \Delta t & 1-\frac{\kappa}{J_{z z} V_{x}} \Delta t & 0 & 0 \\
-\frac{h \sigma}{J_{x x}} \Delta t & \frac{h \rho}{V_{x} J_{x x}} \Delta t & 1-\frac{c}{J_{x x}} \Delta t & \frac{m g h-k}{J_{x x}} \Delta t \\
0 & 0 & \Delta t & 1
\end{array}\right] x(k) \\
& +\left[\begin{array}{c}
\frac{C_{v}}{m V_{\frac{V}{x}}} \Delta J_{x e q} \\
\frac{J_{v x}}{J_{x z}} \Delta t \\
\frac{h C_{v}}{J_{z z}} \Delta t \\
0
\end{array}\right] \delta(k),
\end{aligned}
$$

where $x=\left[\begin{array}{llll}\beta & \dot{\psi} & \dot{\phi} \phi\end{array}\right]^{T}$ is the state vector, $\sigma=C_{v}+C_{h}$, $\rho=C_{v} l_{v}+C_{h} l_{h}, \kappa=C_{v} l_{v}^{2}+C_{h} l_{h}^{2}$ and $J_{x e q}=J_{x x}+m h^{2}$, with the parameters given in Table 1 .

Our aim is to detect the CG change of the given example, applying the proposed structured filter when only the roll angle $\phi$ is measured. Assuming that the CG height of the vehicle can switch between any of the values $h_{1}=$ $0.7, h_{2}=0.8, h_{3}=0.9$ and $h_{4}=1$, we get, respectively, four models $M_{1}, M_{2}, M_{3}$ and $M_{4}$. Therefore, these models are regrouped in two subsets with two models for each of them. Two sub-estimators are developed for the two subsets and the global estimator makes a fusion of the sub-estimators. The expected result is the detection of active mode, using the activation probabilities following the sub-estimator $\mu_{l}^{s_{i}}(k)$ and global estimator $\mu^{s_{i}}(k)$.

We propose a scenario to test and compare the performance of the proposed methods (direct, hierarchical and mixed-decision) with the IMM algorithm. The results of this comparison are presented in Figures 5 and 6.The switches are generated using a Markov matrix $\Pi$ given by

$$
\Pi=\left[\begin{array}{cccc}
0.9 & 0.033 & 0.033 & 0.033 \\
0.033 & 0.9 & 0.33 & 0.033 \\
0.033 & 0.033 & 0.9 & 0.033 \\
0.033 & 0.033 & 0.033 & 0.9
\end{array}\right]
$$

Figure 4 represents the steering angle profile $(\delta(t))$, the output $(\phi(t))$ and the output with noise used in the 
following simulations. Simulations with a noise lead to Fig-ures 5 and 6 where probability activation (in time function) is given for the direct and the hierarchical decision meth-ods. Figure 5 represents the probability activation of each subset and the probability activation of each model calcu-lated by the direct method and those calculated by the IMM method. Figure 6 allows us to compare with the hierarchical decision.

These simulations show that the performances of the proposed detection methods are better than those of the IMM algorithm. Particularly, the hierarchical deci-sion method gives better results compared to the direct decision.

\section{Conclusion}

In this work, a technique of the division of the set of mod-els for the multi-model Bayesian estimators is introduced. Instead of considering only the subset selected in each ex-ecution cycle, the proposed approach considers the set of all models using sub-estimators operating in parallel. Com-pared to conventional multi-model estimators, the struc-tured approach allows acting on new parameters such as the grouping patterns of models in the subsets and the number of subsets. The proposed method also provides additional information such as activation probabilities of subsets and allows two decision levels for active mode. It is applied successfully for the detection of CG change in vehicle dynamics and gives a better result than the clas-sical IMM algorithm. Following this work, a systematic method for grouping models in the subset to exploit the potential of the proposed structure will be developed. More-over, the method will be applied on more complex vehicle models.

\section{References}

Ackermann, J., Bartelett, A., Kaesbauer, D., Sienel, W., \&

Steinhauser, R. (1993). Robust control with uncertain parameters. London: Springer.

Bakker, E., Pacejka, H.B., \& Linder, L. (1989). A new tire model, an application in vehicle dynamics studies. SAE Transactions, 98(6), 101-113.

Bar-Shalom, Y., Chang, K.C., \& Blom, H.A. (1989). Tracking a maneuvering target using input estimation versus the interacting multiple model algorithm. IEEE Transactions on Aerospace and Electronic Systems, 25(2), 296300.

Boers, Y., \& Driessen, J.N. (2005). A multiple model multiple hypothesis filter for Markovian switching systems. Automatica, $41,709-716$.

Dahmani, H., Chadli, M., Rabhi, A., \& El Hajjaji, A. (2013a). Road curvature estimation for vehicle lane departure detection using a robust Takagi-Sugeno fuzzy observer. Vehicle System Dynamics Journal, 51(5), 581-599.
Dahmani, H., Chadli, M., Rabhi, A., \& El Hajjaji, A. (2013b). Vehicle dynamic estimation with road bank angle consideration for rollover detection: Theoretical and experimental studies. Vehicle System Dynamics Journal, 51(12), 18531871.

Dong, H., Wang, Z., \& Gao, H. (2013). Distributed $H_{\infty}$ filtering for a class of Markovian jump nonlinear time-delay systems over lossy sensor networks. IEEE Transactions on Industrial Electronics, 60, 4665-4672.

Dong, H., Wang, Z., Ho, D.W.C., \& Gao, H. (2011). Robust $H_{\infty}$ filtering for Markovian jump systems with randomly occurring nonlinearities and sensor saturation: The finite-horizon case. IEEE Transactions on Signal Processing, 59, 30483057.

Ho, T.-J. (2011). A switched IMM-extended viterbi estimatorbased algorithm for maneuvering target tracking. Automatica, 47, 92-98.

Hocine, A., Chadli, M., Maquin D., \& Ragot J. (2008). A discretetime sliding window observer for Markovian switching system. Control and Intelligent Systems Journa, 36(2), 174-181.

Hocine, A., Maquin, D., \& Ragot, J. (2005). Finite memory observer for switching systems: Application to diagnosis. In Pavel Zítek (Ed.), Proceedings of the 16th IFAC World Congress (pp. 1869-1869). The Prague, Czech Republic.

Karimi, H.R. (2011). Robust delay-dependent $H_{\infty}$ control of uncertain Markovian jump systems with mixed neutral, discrete and distributed time-delays. IEEE Transactions on Circuits and Systems I, 58, 1910-1923.

Li, X.R. (2000). Multiple-model estimation with variable structure. Part II: Model set adaptation. IEEE Transactions on Automatic Control, 45(11), 2047-2060.

Li, X.R., \& Bar-Shalom, Y. (1996). Multiple-model estimation with variable structure Part I. IEEE Transactions on Automatic control, 41(4), 478-493.

Li, X.R., Zhao, Z., \& Li, X.B. (2005). General model-set design methods for multiple-model approach. IEEE Transactions on Automatic Control, 50(9), 1260-1276.

Magill, D.T (1965). Optimal adaptive estimation of sampled stochastic process. IEEE Transactions on Automatic Control, 10(4), 434-439.

Qu, H.Q., Pang, L.P., \& Li, S.H. (2009). A novel interacting multiple model algorithm. Signal Processing, 89, 21712177.

Ryu, J., \& Gerdes, J.C. (2004). Estimation of vehicle roll and road bank angle. In Proceedings of the 2004 American Control Conference. Boston, MA.

Solmaz, S. (2007). Topics in automotive rollover prevention: Robust and adaptive switching strategies for estimation and control (Unpublished PhD thesis). Hamilton Institute of National University of Ireland, Maynooth, Ireland.

Solmaz, S., Shorten, R., Wulff, K., \& O'Cairbre, F. (2008). A design methodology for switched discrete time linear systems with applications to automotive roll dynamics control. Automatica, 44(9), 2358-2363.

Wang, Z, Liu, Y., \& Liu, X. (2010). Exponential stabilization of a class of stochastic system with Markovian jump parameters and mode-dependent mixed time-delays. IEEE Transactions on Automatic Control, 55, 1656-1662.

Wang, Z., Shen, B., \& Liu, X. (2012). $H_{\infty}$ filtering with randomly occurring sensor saturations and missing measurements. Automatica, 48(3), 556-562.

Wei, G., Wang, Z., Shen, B., \& Li, M. (2011). Probabilitydependent gain-scheduled filtering for stochastic systems with missing measurements. IEEE Transactions on Circuits and Systems - Part II, 58(11), 753-757.

Zhang, Y., \& Li, X.R. (1998). Detection and diagnosis of sensor and actuator failures using IMM estimator. IEEE Transactions on Aerospace and Electronic Systems, 34(4), 1293-1312. 